\title{
Critical patch-size for two-sex populations
}

\author{
Gabriel Andreguetto Maciel $^{*, a}$, Renato Mendes Coutinho ${ }^{\mathrm{b}}$, Roberto André Kraenkel ${ }^{\mathrm{c}}$ \\ ${ }^{a}$ Instituto de Física Teórica, Universidade Estadual Paulista - UNESP, R. Dr. Teobaldo Ferraz 271, São Paulo 01140-070, Brazil \\ ${ }^{\mathrm{b}}$ Centro de Matemática, Computação e Cognição, UFABC, Santo André, Brazil \\ ${ }^{\mathrm{c}}$ Instituto de Física Teórica, Universidade Estadual Paulista - UNESP, São Paulo, Brazil
}

\section{A R T I C L E I N F O}

\section{Keywords:}

Two-sex model

Critical patch size

Reaction-diffusion equations

Habitat loss

\begin{abstract}
A B S T R A C T
As environments become increasingly degraded, mainly due to human activities, species are often subject to isolated habitats surrounded by unfavorable regions. Since the pioneering work by Skellam [25] mathematical models have provided useful insights into the population persistence in such cases. Most of these models, however, neglect the sex structure of populations and the differences between males and females. In this work we investigate, through a reaction-diffusion system, the dynamics of a sex-structured population in a single semipermeable patch. The critical patch size for persistence is determined from implicit relationships between model parameters. The effects of the various growth and movement parameters are also investigated.
\end{abstract}

\section{Introduction}

Population dynamics studies usually assume that a species is characterized by a single sex-class of individuals, usually females. This approach is surely appropriate for hermaphrodite species and in systems where population dynamics is determined by a single limiting sex [24]. However, as in many species males and females have quite distinct demographic parameters and no sex dominates population dynamics, these unstructured models become unrealistic and models that take into account the dynamics of both sexes are needed [4].

Males and females can show, for instance, quite distinct dispersal abilities, which can have a great impact on their spatial distributions. To cite a few examples, dispersal of $C$. carcharias (white shark) is sexbiased with philopatric (non-roving) females and roving males [21]; at local scale, dispersal in the common vole $M$. arvalis is strongly malebiased [10]. It has been suggested as a general result that in mammals males usually disperse more frequently than females, while in birds females are the most dispersive sex [11]. In human-altered environments sex-biased dispersal is thought to expose populations to an even greater risk of extinction [6]. Individuals that used to disperse to other sites in the landscape may not find suitable areas, resulting in increased loss of the most dispersive gender. As a consequence, sex-biased dispersal may lead to biased sex-ratios in the natal population, leaving a higher number of individuals unpaired.

Despite the increasing number of empirical studies on sex-related effects, very few mathematical studies have analyzed the dynamics of sex-structured populations in space. Miller et al. [20] derived an explicit expression for the speed of invasion of a two-sex integrodifference model. The effect of sex-biased dispersal on the spreading speed was then studied. Reaction-diffusion equations have also been used to study the dynamics of sex-structured populations in space $[1,14]$. Jin et al. [14] modeled populations with short reproductive seasons in patches of limited sizes through a periodic impulsive reaction-diffusion system with Dirichlet boundary conditions, and identified conditions for population persistence.

Increasing degradation of natural areas frequently subject populations to habitats of limited size surrounded by unfavorable regions. Since the pioneering work by Skellam [25], reaction-diffusion equations have become one of the most important mathematical tools in the study of population persistence in such situations. Skellam posed the "minimal size problem" of how large a patch must be to support a population. For a single population with hostile boundaries, diffusion constant $d$ and intrinsic growth rate $r$, the critical size of a one-dimensional patch is $l_{c}=\pi \sqrt{d / r}$.

In this study we examine the minimal size problem for sex-structured populations. We model population dynamics through a reactiondiffusion system with reproduction given by the standard harmonic mean mating function $[16,19]$. We study the problem of species persistence in a finite domain with semipermeable boundaries and show how standard techniques in the analysis of monotone parabolic partial differential equations can be used to find an implicit relation that determines the critical patch size. In the limit of completely hostile boundary conditions, this critical patch size can be expressed explicitly in terms of model parameters. Moreover, we study how distinct males' and females' traits affect the critical patch size.

\footnotetext{
* Corresponding author.

E-mail address: gabriel.andreguetto@gmail.com (G. Andreguetto Maciel).
} 


\section{The model}

Growth in two-sex models is a process that involves pair formation and the production of new offspring by females. The number of couples for certain local female $(f)$ and male $(m)$ populations is given by the mating (or birth) function $\mathfrak{B}(m, f)$. Several forms for $\mathfrak{B}$ have been proposed $[12,20]$. This function is usually required to follow some desired properties [3,12]: it needs to be a positive, non-decreasing function for all $m, f \neq 0$, as well as homogeneous, that is, $\mathfrak{B}(a m, a f)=a \mathfrak{B}(m, f)$ for all $a \neq 0$. The harmonic mean function

$\mathfrak{B}(m, f)=\frac{2 m f}{m+f}$

follows all these properties and is free from the difficulties other functions present [15]. The geometric mean $\mathfrak{B}(m, f)=\sqrt{m f}$ and minimum function $\mathfrak{B}(m, f)=\min (m, f)$ are other common mating functions. The harmonic mean mating function is often considered the least objectionable mating function [16]. Also, Miller and Inouye [19] verified in a laboratory experiment that the harmonic mean mating function best describes the growth of the bean beetle (Callosobruchus maculatus) at low densities.

$\mathfrak{B}$ can be easily modified to account for polygamous systems, in which males or females can mate with more than one partner. If $h$ represents the number of females with which each male mates, also known as the "harem" size, the number of "harems" formed is given by $\mathfrak{B}(m, f / h)$ [24]. The number of paired females is then given by $h \mathfrak{B}(m, f / h)$. Polygynous, monogamous and polyandrous mating systems can be accommodated by setting $h>1, h=1$ or $h<1$, respectively.

Now we assume that individuals of both sexes move and reproduce in a single one-dimensional habitat of size $l$. The local number of reproducing females for given male $(m)$ and female $(f)$ densities is assumed to be given by the harmonic mean mating function, Eq. (1). Different mating systems are accommodated by properly introducing the harem size $(h)$ into the model, as described above. We also assume that male and female diffusivities, $d_{m}$ and $d_{f}$ respectively, are distinct in general. Denoting time as $t \geq 0$ and spatial positions by $x \in[-l / 2, l / 2]$, we write the reaction-diffusion system of equations for male and female populations as:

$\frac{\partial m}{\partial t}=d_{m} \frac{\partial^{2} m}{\partial x^{2}}+c g \frac{2 m f}{m+f / h}-\mu_{m} m$

$\frac{\partial f}{\partial t}=d_{f} \frac{\partial^{2} f}{\partial x^{2}}+(1-c) g \frac{2 m f}{m+f / h}-\mu_{f} f$

where $c \in[0,1]$ is the fraction of males at birth and $g$ is the rate of production of new offsprings per paired female, while $\mu_{m}$ and $\mu_{f}$ are the male and female mortality rates, respectively.

At patch boundaries we impose semipermeable boundary conditions $[5,27]$. We initially write these conditions in the form given by Van Kirk and Lewis [27]:

$\frac{\partial m}{\partial x}( \pm l / 2)=\mp c_{m} m( \pm l / 2)$

$\frac{\partial f}{\partial x}( \pm l / 2)=\mp c_{f} f( \pm l / 2)$

where parameters $c_{m, f} \geq 0$ represent the enticement of individuals to leave the patch. For $c_{m}=0\left(c_{f}=0\right)$ no males (females) leave the patch and we get no-flux conditions. In the limit $c_{m, f} \rightarrow \infty$, individuals leave the patch and never return.

We introduce the new scaled quantities:

$$
\begin{aligned}
M=m, \quad F & =\frac{f}{h}, \quad T=g t, \quad X=\sqrt{\frac{g}{d_{m}}} x, \quad \tilde{\mu}_{m, f}=\frac{\mu_{m, f}}{g}, \\
D & =\frac{d_{f}}{d_{m}}, \quad L=\sqrt{\frac{g}{d_{m}}} l,
\end{aligned}
$$

and rewrite the equations as:

$\frac{\partial M}{\partial T}=\frac{\partial^{2} M}{\partial X^{2}}+c \frac{2 h M F}{M+F}-\tilde{\mu}_{m} M$

$\frac{\partial F}{\partial T}=D \frac{\partial^{2} F}{\partial X^{2}}+(1-c) \frac{2 M F}{M+F}-\tilde{\mu}_{f} F$.

In this new scaling we can define the boundary permeability of males and females as

$\alpha_{m, f}=\frac{c_{m, f} \sqrt{\frac{d_{m}}{g}}}{\left(1+c_{m, f} \sqrt{\frac{d_{m}}{g}}\right)}$.

Boundary conditions then read:

$\left(1-\alpha_{m}\right) \frac{\partial M}{\partial X}( \pm L / 2)=\mp \alpha_{m} M( \pm L / 2)$

$\left(1-\alpha_{f}\right) \frac{\partial F}{\partial X}( \pm L / 2)=\mp \alpha_{f} F( \pm L / 2)$.

Parameters $\alpha_{m, f} \in[0,1]$ control boundary permeability and can represent both individual movement decisions [27] and the quality of the exterior region [17]. In the limit $\alpha_{m, f}=0$ no individuals are lost through the boundaries and we get no-flux conditions. Yet when $\alpha_{m, f}=1$ we get hostile boundary conditions. In this case, every individual that hits a boundary leaves the patch and never returns.

The Eqs. (6)-(7) form a system of nonlinear partial differential equations that is homogeneous, in the sense that if $(M(X, T), F(X, T))$ is a solution to the system, then $(s M(X, T), s F(X, T))$, with $s$ a given constant, is a solution as well. Note also that there is no saturating mechanism in our mating function and therefore solutions either decrease to zero or grow to infinity asymptotically. Accordingly, our model is appropriate to investigate the growth of small populations and specifically the critical size problem.

\section{Results}

Our goal is to derive persistence conditions for our sex-structured model, i.e. conditions for which the population can grow. Persistence conditions for problems without Allee effects are typically derived from the linear stability of the trivial zero steady state solution [2]. When we try to linearize Eqs. (6)-(7) around the $(0,0)$ state, though, we obtain an indeterminacy in the sex-ratio of populations, $M / F=0 / 0$, which leads to the mating function being undefined. In what follows below, we obtain conditions for species persistence instead by finding particular exponential solutions for this system and determining conditions for the growth of these solutions. Using results from maximum principles for parabolic partial differential equations, we then show that these conditions imply persistence of the population for a general class of initial conditions.

\subsection{Particular solutions}

We seek solutions of the form:

$M(X, T)=M_{0} e^{\lambda T} V(X)$

$F(X, T)=F_{0} e^{\lambda T} V(X)$,

where $M_{0}$ and $F_{0}$ are given positive constants. As population densities are always positive, we consider only real values of $\lambda$, and $V(X) \geq 0$. Substituting these expressions into Eqs. (6) and (7), $V(X)$ must satisfy 
simultaneously the two equations:

$\frac{d^{2} V}{d X^{2}}+\delta_{m}^{2} V=0$

$\frac{d^{2} V}{d X^{2}}+\delta_{f}^{2} V=0$,

where:

$\delta_{m}^{2}=\frac{2 h c}{1+\sigma_{0}}-\tilde{\mu}_{m}-\lambda$

$\delta_{f}^{2}=\frac{1}{D}\left[\frac{2(1-c) \sigma_{0}}{1+\sigma_{0}}-\tilde{\mu}_{f}-\lambda\right]$.

In the above equations $\sigma_{0}=M_{0} / F_{0}$. Note that $V(X)$ must also satisfy boundary conditions (8)-(9).

Solutions for Eq. (12) with negative $\delta_{m}^{2}$ lead to real-valued exponential functions that do not satisfy the boundary conditions (8). Therefore, $\delta_{m}$ is real and can be assumed non-negative without loss of generality, so the solution of (12) can be written as:

$V(X)=P \sin \left(\delta_{m} X\right)+Q \cos \left(\delta_{m} X\right)$

where $P$ and $Q$ are constants to be determined. Applying conditions (8), $P$ and $Q$ are obtained from the solution of the linear system:

$\mathbf{A}\left[\begin{array}{l}P \\ Q\end{array}\right]=\left[\begin{array}{cc}A & B \\ A & -B\end{array}\right]\left[\begin{array}{l}P \\ Q\end{array}\right]=0$,

with $A$ and $B$ given by:

$A=\left(1-\alpha_{m}\right) \delta_{m} \cos \left(\delta_{m} L / 2\right)+\alpha_{m} \sin \left(\delta_{m} L / 2\right)$

$B=-\left(1-\alpha_{m}\right) \delta_{m} \sin \left(\delta_{m} L / 2\right)+\alpha_{m} \cos \left(\delta_{m} L / 2\right)$

System (17) admits nontrivial solutions only if $\operatorname{det}(\mathbf{A})=0$, so either $A$ or $B$ is null. Note that if $A$ and $B$ are both null we must have $\delta_{m}^{2}=-\left[\alpha_{m} /\left(1-\alpha_{m}\right)\right]^{2}$ contradicting the required positivity of $\delta_{m}^{2}$ (see above). If $A=0$ (and hence $B \neq 0$ ), we must have $Q=0$ and therefore: $V(X)=P \sin \left(\delta_{m} X\right)$. This solution, however, changes sign at the origin. By the positivity of $V$, we must have $A \neq 0$, and therefore $B=0$. The analysis for female Eq. (13) with boundary conditions (9) is completely analogous. Positive solutions of the form (10)-(11) thus require:

$\tan \left(\frac{\delta_{m} L}{2}\right)=\frac{\alpha_{m}}{1-\alpha_{m}} \frac{1}{\delta_{m}}$

$\tan \left(\frac{\delta_{f} L}{2}\right)=\frac{\alpha_{f}}{1-\alpha_{f}} \frac{1}{\delta_{f}}$

Since $\delta \tan (\delta L / 2)$ spans the whole $\mathbb{R}^{+}$for $\delta \in[0, \pi / L)$ and $\alpha /(1-\alpha) \in \mathbb{R}^{+}$, each equation above always has a real root for $\delta_{m, f}$ in the interval $[0, \pi / L)$. This guarantees that, with this choice of $\delta_{m}, V(X)$ is indeed positive in the domain $[-L / 2, L / 2]$. On the other hand, Eqs. (14) and (15) yield $\lambda$ and $\sigma_{0}$ in terms of $\delta_{m}$ and $\delta_{f}$ :

$\sigma_{0}=\frac{2 h c-\tilde{\mu}_{m}-\lambda-\delta_{m}^{2}}{\tilde{\mu}_{m}+\lambda+\delta_{m}^{2}}$

$\lambda=\frac{2 h c(1-c)-(1-c)\left(\tilde{\mu}_{m}+\delta_{m}^{2}\right)-h c\left(\tilde{\mu}_{f}+D \delta_{f}^{2}\right)}{h c+(1-c)}$.

Thus, for each set of parameters, the possible values of $\lambda$ are determined by transcendental Eqs. (20) and (21). Growth or decline of the population is then determined by the sign of the largest $\lambda\left(\lambda^{*}\right)$.

Eqs. (20)-(21) present an infinite number of solutions but, since (23) is decreasing in both $\delta_{m}^{2}$ and $\delta_{f}^{2}, \lambda *$ is determined by the smallest values of $\delta_{m}$ and $\delta_{f}$ (assumed both positive), that is, the smallest positive roots of Eqs. (20)-(21). In the following subsection we show that $\lambda *$ also determines the growth of general initial conditions.

\subsection{Outcomes of general initial conditions}

We begin by showing that the system (6)-(7) preserves the order of solutions. This follows from the application of maximum principles for parabolic pdes $[2,22]$.

We write the system of Eqs. (6), (7) as:

$\frac{\partial M}{\partial T}-\frac{\partial^{2} M}{\partial X^{2}}=G_{M}(M, F)$

$\frac{\partial F}{\partial T}-D \frac{\partial^{2} F}{\partial X^{2}}=G_{F}(M, F)$

where

$$
\begin{aligned}
G_{M}(M, F)= & c \frac{2 h M F}{M+F}-\tilde{\mu}_{m} M \quad \text { and } \quad G_{F}(M, F)=(1-c) \frac{2 M F}{M+F} \\
& -\tilde{\mu}_{f} F
\end{aligned}
$$

This system is cooperative as it can be readily seen that $\frac{\partial G_{M}}{\partial F}>0$ and $\frac{\partial G_{F}}{\partial M}>0$. In addition, $G_{M}(M, F)$ and $G_{F}(M, F)$ are continuous functions with continuous first derivatives of their variables.

With these properties established, along with boundary conditions $(8,9)$, this system satisfies the hypotheses of the comparison theorem 1.20 in [2]. This theorem implies that, given two solutions $S_{2}=\left(M_{2}(X, T), F_{2}(X, T)\right)$ and $S_{1}=\left(M_{1}(X, T), F_{1}(X, T)\right)$ of $(6,7)$ subject to boundary conditions $(8,9)$ such that initially we have

$M_{2}(X, 0) \geq M_{1}(X, 0)$

$F_{2}(X, 0) \geq F_{1}(X, 0)$

in $\Omega=\{X \in(-L / 2, L / 2)\}$ and either

$M_{2}( \pm L / 2, T) \geq M_{1}( \pm L / 2, T)$

$F_{2}( \pm L / 2, T) \geq F_{1}( \pm L / 2, T)$

or

$\alpha_{m} M_{2}( \pm L / 2, T) \pm\left(1-\alpha_{m}\right) \frac{\partial M_{2}}{\partial X}( \pm L / 2, T) \geq \alpha_{m} M_{1}( \pm L / 2, T) \pm\left(1-\alpha_{m}\right) \frac{\partial M_{1}}{\partial X}( \pm L / 2, T)$

$\alpha_{f} F_{2}( \pm L / 2, T) \pm\left(1-\alpha_{f}\right) \frac{\partial F_{2}}{\partial X}( \pm L / 2, T) \geq \alpha_{f} F_{1}( \pm L / 2, T) \pm\left(1-\alpha_{f}\right) \frac{\partial F_{1}}{\partial X}( \pm L / 2, T)$,

for $T \in(0, \bar{T}]$, where $\bar{T}>0$ is any given instant of time, then

$M_{2}(X, T) \geq M_{1}(X, T)$

$F_{2}(X, T) \geq F_{1}(X, T)$ in $\Omega \times(0, \bar{T}]$,

and the ordering of solutions is maintained.

We use this ordering property to show that the largest $\lambda$ in (23), $\lambda *$, also determines the growth or decline of solutions to the system evolving from general initial conditions. Suppose that for a given set of parameters we have $\lambda^{*}>0$, so that solutions (10)-(11) grow with time. Given initial conditions $M(X, 0)$ and $F(X, 0)$ that are strictly positive in $\Omega, M_{0}$ and $F_{0}$ can always be taken small enough in a way that $M(X$, $0) \geq M_{0} V(X)$ and $F(X, 0) \geq F_{0} V(X)$ for $X \in \Omega$. As the solutions evolved from these initial conditions satisfy boundary conditions, conditions (30)-(31) are clearly satisfied. Thus, the ordering of solutions is preserved and it follows that solutions obtained from initial conditions $M$ $(X, 0)$ and $F(X, 0)$ also increase with time. In a similar way, if $\lambda *<0$ we can choose $M_{0}$ and $F_{0}$ large enough so that $M(X, 0) \leq M_{0} V(X)$ and $F(X$, $0) \leq F_{0} V(X)$ in $\Omega$. In this case the decline of solutions (10)-(11) imply the decline of general solutions.

At first, the reasoning above does not apply for initial conditions that have zeros in the domain, as in this case the only way of bounding these functions below by initial conditions of (10)-(11) is making $M_{0}=F_{0}=0$. This case, however, can be accommodated by applying the 
following basic result from maximum principles.

The system of Eqs. (6), (7) can be rewritten as:

$$
\begin{aligned}
& \frac{\partial M}{\partial T}-\left[\frac{\partial^{2} M}{\partial X^{2}}-\tilde{\mu}_{m} M\right]=c \frac{2 h M F}{M+F} \geq 0 \\
& \frac{\partial F}{\partial T}-\left[D \frac{\partial^{2} F}{\partial X^{2}}-\tilde{\mu}_{f} F\right]=(1-c) \frac{2 M F}{M+F} \geq 0 .
\end{aligned}
$$

Given the conditions above, the maximum principle (see Theorem 1.17 in [2]) states that if $M(F)$ attains a minimum value $M_{\min } \leq 0\left(F_{\min } \leq 0\right)$ at a point $\left(X_{0}, T_{0}\right)$ in $\Omega \times(0, \bar{T}]$, then $M(X, T) \equiv M_{\min }\left(F(X, T) \equiv F_{\min }\right)$ on $\Omega \times\left(0, T_{0}\right]$. This shows that any solution to our system with initial conditions that contains zeros in the domain becomes strictly positive at $T>0$ and the reasoning described earlier applies. We have thus shown that persistence in this system is in general determined by the sign of $\lambda *$.

\subsection{Critical patch sizes}

We now investigate the effects of the various parameters on the critical patch size, $L_{c}$. If $L<L_{c}, \lambda^{*}<0$ and the population is eliminated. For $L>L_{c}$ we have $\lambda^{*}>0$ and the population can grow. We compute $L_{c}$ by numerically determining the patch size for which $\lambda^{*}$ changes sign. As $\lambda$ is decreasing in both $\delta_{m}^{2}$ and $\delta_{f}^{2}$ we use the smallest roots of (20) and (21), to compute $\lambda^{*}$ from (23).

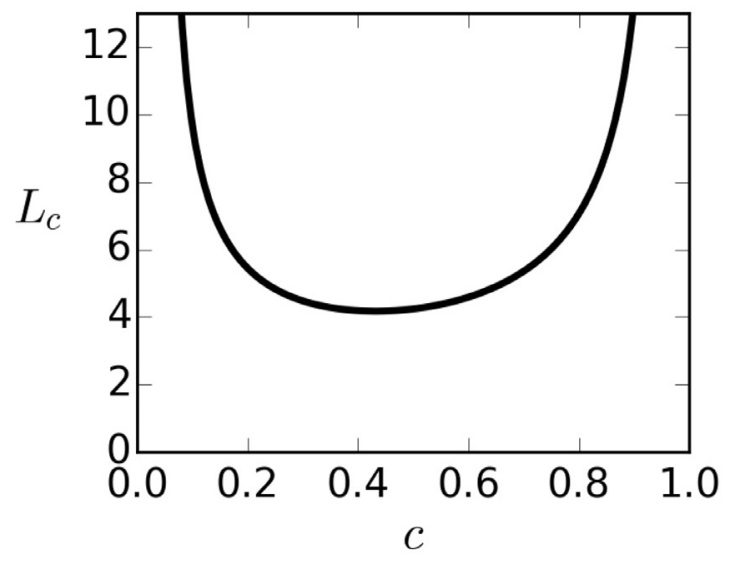

(a)

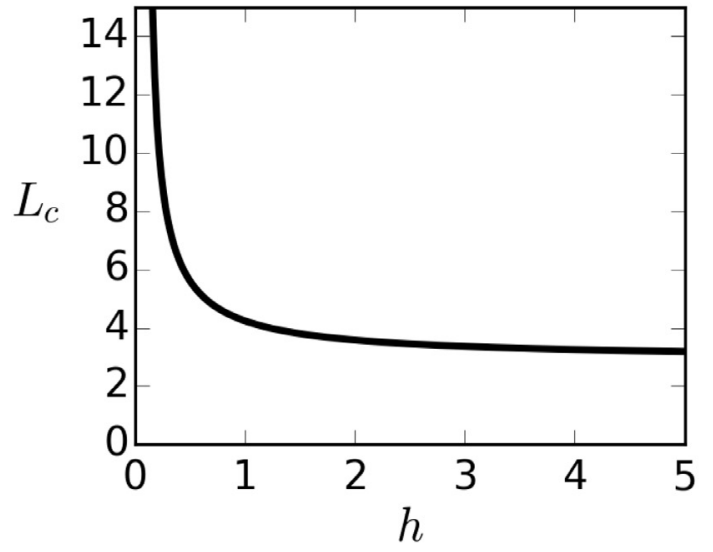

(c)
Fig. 1a shows the critical patch size as a function of the fraction of males at birth, $c$. $L_{c}$ is minimized at intermediate fraction $c$. As we have set $D=2$ (so $d_{f}=2 d_{m}$ ), the minimum occurs for a ratio lower than half. Thus, persistence is enhanced when the more dispersive sex, females in this case, has a larger fraction of individuals at birth. The critical patch size is an increasing function of the mortality rates of both populations (see Fig. 1b) and a decreasing function of "harem size" (see Fig. 1c).

When $h$ becomes very large, the dynamics is completely determined by females. The critical patch size is then given by the single species threshold $[5,17]$ :

$L_{c}=2 \sqrt{\frac{D}{2(1-c)-\tilde{\mu}_{f}}} \arctan \left(\frac{\alpha_{f}}{1-\alpha_{f}} \sqrt{\frac{D}{2(1-c)-\tilde{\mu}_{f}}}\right)$.

This expression is obtained by taking the limit $h \rightarrow \infty$ and $\lambda=0$ in Eq. (23), from which we determine $\delta_{f}$. $L_{c}$ is then obtained after substituting the expression for $\delta_{f}$ in (21). Note that (36) corresponds to the critical patch size of a single population with diffusion constant $D$ and intrinsic growth rate $r=2(1-c)-\tilde{\mu}_{f}$, subject to boundary conditions (9). The effect of the scaled diffusivity, $D$, is shown in Fig. 1d. The critical patch size increases with the diffusivity of females.

Finally, Fig. 2a-c illustrate how boundary permeabilities affect $L_{c}$. In panel Fig. 2a female permeability $\alpha_{f}$ is kept constant while we change $\alpha_{m}$. In panel Fig. 2b we do the reverse. Both permeabilities are varied in panel Fig. 2c maintaining them equal to each other. As expected, the increase of permeability of males and females increases the critical

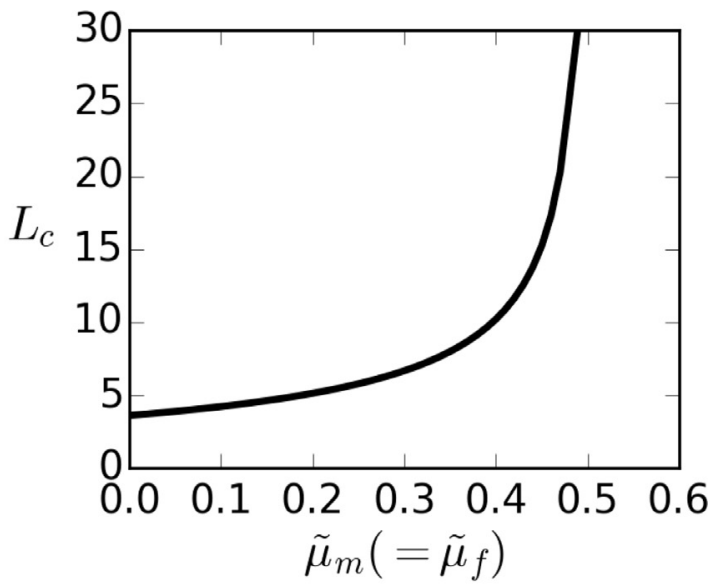

(b)

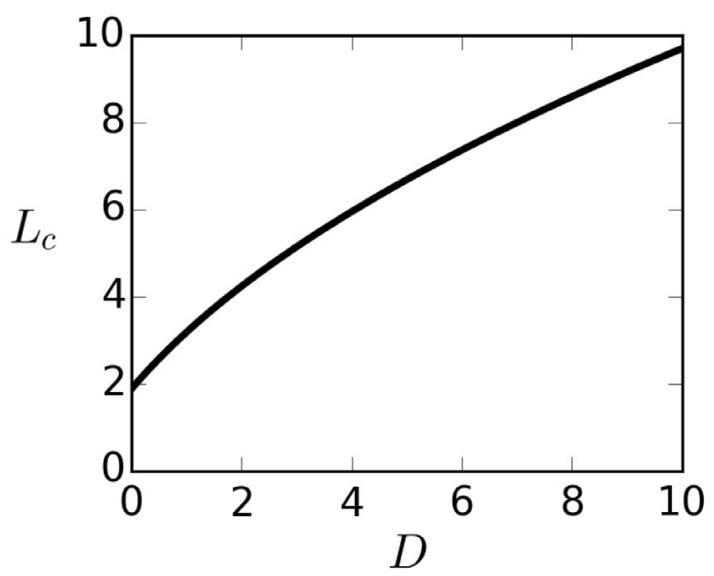

(d)

Fig. 1. Critical patch size as a function of: (a) fraction of males at birth, (b) mortality rates, (c) "harem size" and (d) the scaled diffusivity $D$. Except when being varied, we use the parameter values: $D=2, c=0.5, h=1$ and $\widetilde{\mu}_{m}=\widetilde{\mu}_{f}=0.1$. In all panels $\alpha_{m}=\alpha_{f}=0.5$. 


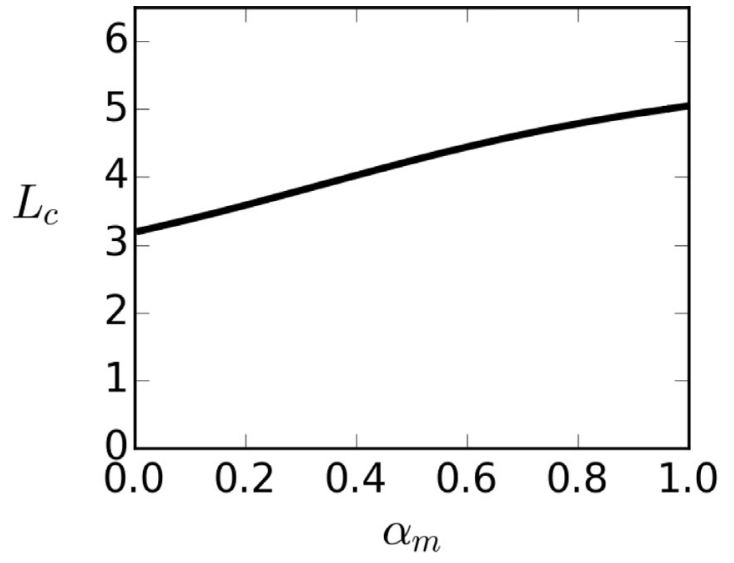

(a)

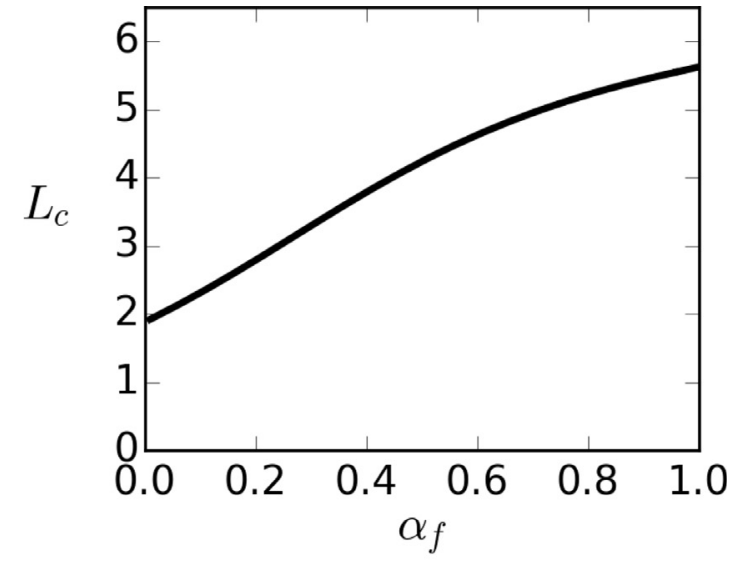

(b)

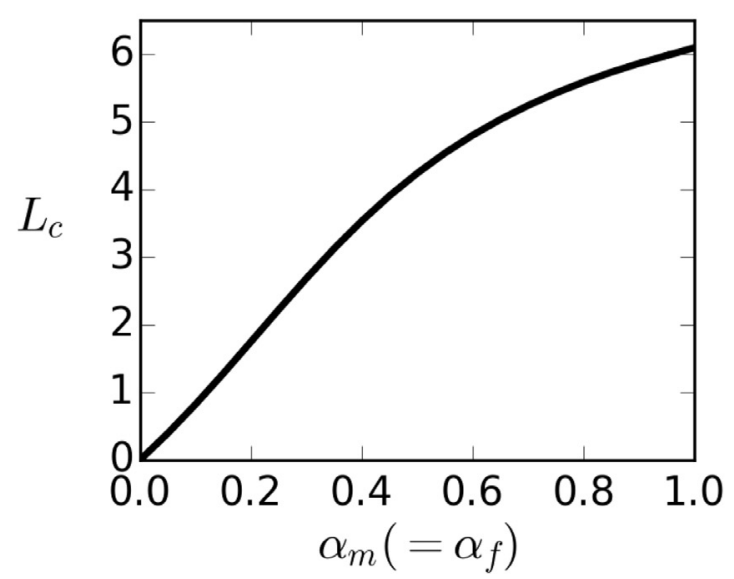

(c)

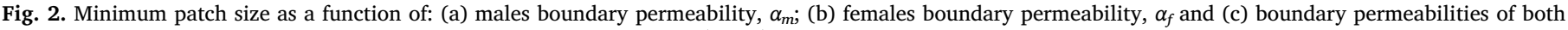
sexes while keeping $\alpha_{m}=\alpha_{f}$. Parameters are: $D=2, c=0.5, h=1$ and $\tilde{\mu}_{m}=\widetilde{\mu}_{f}=0.1$. We have $\alpha_{f}=0.5$ in (a) and $\alpha_{m}=0.5$ in (b).

patch size. Because we have set the female diffusion rate larger than for males $(D=2)$, the female permeability has a stronger effect on $L_{c}$ when varied alone (see Fig. 2b).

\subsection{Hostile boundary conditions}

Completely hostile boundary conditions for males and females are obtained when $\alpha_{m}=\alpha_{f}=1$. In this limit population densities are null at boundaries and every individual that hits a boundary leaves the patch and never returns. We show below that an explicit expression for the critical patch size can be obtained in this case.

Taking the limit $\alpha_{m}=\alpha_{f} \rightarrow 1$ in Eqs. (20) and (21) yields that the first (smallest) positive root $\delta_{m}\left(\delta_{f}\right)$ tends to $\pi / L$, which corresponds to the first asymptote of the tangent function. Plugging this result into (23) and imposing the criticality condition $\lambda=0$, we obtain the following critical patch size:

$L_{c}=\pi \sqrt{\frac{(1-c)+h c D}{2 h c(1-c)-(1-c) \tilde{\mu}_{m}-h c \tilde{\mu}_{f}}}$.

\section{Discussion}

The differences between male's and female's traits are often neglected in population dynamics studies. On the other hand, the importance of sex-specific characteristics and the role of males in population growth have been increasingly acknowledged [23]. In this study we analyzed a two-sex model in a single patch with semipermeable boundaries. Finding particular solutions and using maximum principles for parabolic partial differential equations, we derived implicit expressions for the critical patch size. As far as we know, this study provides the first derivation of the critical patch size of a two-sex model with continuous movement and reproduction.

Our results show that the qualitative dependence of the critical patch size on the parameters of the model matches the biological expectations. $L_{c}$ is minimized at intermediate fraction of males at birth and grows infinitely large as mortality rates increase. The minimum size of the patch is a decreasing function of the "harem" size $h$ and an increasing function of the scaled female diffusivity $D$. Highly permeable patches - large $\alpha_{m}$ and $\alpha_{f}$-require larger patch sizes for the population to persist.

We have limited our analysis to the case of a harmonic mean mating function (Eq. (1)). Surely, this mating function does not accomodate all the rich variety of mating systems encountered in nature. However, it follows all the desired properties of a mating function and has been widely applied in the ecological literature $[15,16,20]$. It is therefore a suitable choice to study the basic effects of space limitation on sexstructured populations. From a mathematical perspective, this function allowed us to obtain particular solutions on the form (10)-(11) and simple relations for the critical patch size. 
A closer inspection on the equations reveals that as long as the mating function is homogeneous (i.e. $\mathfrak{B}(a m, a f)=a \mathfrak{B}(m, f)$ ) particular solutions can be found. For instance, in the model

$\frac{\partial m}{\partial t}=d_{m} \frac{\partial^{2} m}{\partial x^{2}}+c g \mathfrak{B}(m, f)-\mu_{m} m$

$\frac{\partial f}{\partial t}=d_{f} \frac{\partial^{2} f}{\partial x^{2}}+(1-c) g \mathfrak{B}(m, f)-\mu_{f} f$,

the ansatz $m(x, t)=m_{0} e^{\lambda t} v(x), f(x, t)=f_{0} e^{\lambda t} v(x)$ leads to

$\frac{\partial^{2} v}{\partial x^{2}}+\delta_{m}^{2} v=0$

$\frac{\partial^{2} v}{\partial x^{2}}+\delta_{f}^{2} v=0$

where

$\delta_{m}^{2}=\left[\frac{\operatorname{cg} \mathfrak{B}\left(\sigma_{0}, 1\right)}{\sigma_{0}}-\mu_{m}-\lambda\right] \frac{1}{d_{m}}$

$\delta_{f}^{2}=\left[(1-c) g \mathfrak{B}\left(\sigma_{0}, 1\right)-\mu_{f}-\lambda\right] \frac{1}{d_{f}}, \quad \sigma_{0}=m_{0} / f_{0}$.

Similarly as in Eqs. (22) and (23), we can solve (42) and (43) for $\lambda$ and $\sigma_{0}$ to determine persistence conditions. Our procedure therefore is applicable, in principle, to a general class of homogeneous mating functions. For other forms of $\mathfrak{B}(m, f)$, however, the determination of $\sigma_{0}$ from (42) and (43) may not be as simple as in the harmonic mean case.

In [1] the following non-homogeneous (i.e. $\mathfrak{B}(a m, a f) \neq a \mathfrak{B}(m, f)$ ) mating function was derived:

$\mathfrak{B}(m, f)=\frac{\rho m f}{1+\rho T_{c}(m+f)}$,

where $\rho$ is the search rate and $T_{c}$ is the coupling time. If this mating function is used in Eqs. (2), (3), instead of the harmonic mean, a bistable behavior is introduced in the system. Then, when persistence is possible, either elimination or the unbounded growth of both sexes are obtained, depending on the initial conditions. In this case the notion of a critical patch size is slightly different. A well-defined question is if there exists a patch size below which even an infinitely large initial population would go extinct. We argue that, since function (44) corresponds to the harmonic mean when populations are large, this critical size corresponds to $L_{c}$ derived from our model, after a redefinition of constants.

Another related aspect of spatial population dynamics which has been poorly investigated in sex structured models is the speed of spatial spread. Miller et al. [20] obtained the speed of invasion of a two-sex integrodifference model in homogeneous space. Ashih and Wilson [1] studied a two-sex reaction-diffusion model with two female classes, unpaired and fertilized, and calculated the rate of spatial spread in the limit of low gestation times. We are not aware of any calculation of the invasion speed for the basic two-sex reaction-diffusion model given by Eqs. (2), (3). The assumption of traveling wave solutions $M=M_{0} V(X-C T)$ and $F=F_{0} V(X-C T)$ is only compatible with no dispersal bias (results not shown), $D=1$. As a matter of fact, the difference between diffusivities produces a spatially dependent sex-ratio at the leading edge of invasion and the assumption of a constant $\sigma=M / F=M_{0} / F_{0}$ no longer holds. These questions are left for further studies.

In our analysis, the population of each sex was considered homogeneous and therefore a homogeneous mixing between males and females was assumed. The subdivision of populations in different subgroups and the consideration preferential mixing between the subgroups have shown to induce major effects on the dynamics of epidemiological models [7-9]. We conjecture that the inclusion of such effects can affect the critical-patch size of two-sex populations.

The development of spatial two-sex population models is still in its beginnings. As new empirical studies on the dispersal of sex-structured populations emerge $[13,26]$, new models that incorporate greater realism will be needed. The consideration of other spatial arrangements, for example, can bring a better connection to empirical results. Our contribution paves the way for a broader understanding of sex-related effects. Finally, we suggest that in a space composed of many patches, consideration of sex-specific edge behavior by using the framework presented in [18] may reveal interesting results.

\section{Disclosure statement}

The authors declare that they have no conflicts of interest.

\section{Acknowledgments}

GAM and RAK thank CNPq (Brazil) for partial support. GAM has been supported by phd grant \#2011/08166-0, São Paulo Research Foundation (FAPESP). RMC has been supported by post-doctoral grant \#2014/23497-0, São Paulo Research Foundation (FAPESP).

\section{References}

[1] A.C. Ashih, W.G. Wilson, Two-sex population dynamics in space: effects of gestation time on persistence, Theor. Popul. Biol. 60 (2001) 93-106.

[2] R.S. Cantrell, C. Cosner, Spatial Ecology via Reaction-Diffusion Equations, 1st, Wiley, 2003.

[3] H. Caswell, Matrix Population Models: Construction, Analysis, and Interpretation, 2nd, Sinauer, 2001.

[4] H. Caswell, D.E. Weeks, Two-sex models: chaos, extinction, and other dynamic consequences of sex, Am. Nat. 128 (5) (1986) 707-735.

[5] C.A. Cobbold, F. Lutscher, Mean occupancy time: linking mechanistic movement models, population dynamics and landscape ecology to population persistence, J. Math. Biol. 68 (3) (2014) 549-579.

[6] S. Dale, Female-biased dispersal, low female recruitment, unpaired males, and the extinction of small and isolated bird populations, Oikos 92 (2001) 344-356.

[7] S.Y. Del Valle, J.M. Hyman, N. Chitnis, Mathematical models of contact patterns between age groups for predicting the spread of infectious diseases, Math. Biosci. Eng. 10 (2013) 1475-1497.

[8] F.T. Denton, B.G. Spencer, Immigration and the rate of population mixing: explorations with a stylized model, IZA J. Dev. Migr. 7 (2017) 13.

[9] Z. Feng, A.N. Hill, P.J. Smith, J.W. Glasser, An elaboration of theory about preventing outbreaks in homogeneous populations to include heterogeneity or preferential mixing, J. Theor. Biol. 386 (2015) 177-187.

[10] B. Gauffre, E. Petit, S. Brodier, V. Bretagnolle, J.F. Cosson, Sex-biased dispersal patterns depend on the spatial scale in a social rodent, Proc. R. Soc. B 276 (2009) 3487-3494.

[11] P.J. Greenwood, Mating systems, phylopatry and dispersal in birds and mammals, Anim. Behav. 28 (1980) 1140-1162.

[12] K.P. Hadeler, Pair formation in age-structured populations, Acta Appl. Math. 14 (1989) 91-102

[13] K.A. Harrisson, A. Pavlova, J.N. Amos, J.Q. Radford, P. Sunnucks, Does reduced mobility through fragmented landscapes explain patch extinction patterns for three honeyeaters? J. Anim. Ecol. 83 (2014) 616-627.

[14] W. Jin, H.L. Smith, H.R. Thieme, Persistence and critical domain size for diffusing populations with two sexes and short reproductive season, J. Dyn. Differ. Equ. 28 (3) (2016) 689-705.

[15] N. Keyfitz, The mathematics of sex and marriage, Proceedings of the 6th Berkeley Symposium of Mathematical Statistics and Probability, 4 (1972), pp. 89-108.

[16] M. Kot, Elements of Mathematical Ecology, Cambridge University Press, Cambridge, 2001.

[17] D. Ludwig, D.G. Aronson, H.F. Weinberger, Spatial patterning of the spruce budworm, J. Math. Biol. 8 (1979) 217-258.

[18] G.A. Maciel, F. Lutscher, How individual movement response to habitat edges affects population persistence and spatial spread, Am. Nat. 182 (1) (2013) 42-52.

[19] T.E.X. Miller, B.D. Inouye, Confronting two-sex demographic models with data, Ecology 92 (11) (2011) 2141-2151.

[20] T.E.X. Miller, A.K. Shaw, B.D. Inouye, M.G. Neubert, Sex-biased dispersal and the speed of two-sex invasions, Am. Nat. 177 (5) (2011) 549-561.

[21] A.T. Pardini, C.S. Jones, L.R. Noble, B. Kreiser, H. Malcolm, B.D. Bruce, J.D. Stevens, G. Cliff, M.C. Scholl, M. Francis, C.A. Duffy, A.P. Martin, M. Pascoal, 
Sex-biased dispersal of great white sharks, Nature 412 (2001) 139-140.

[22] M.H. Protter, H.F. Weinberger, Maximum Principles in Differential Equations, Prentice-Hall, Englewood Cliffs, NJ, 1967.

[23] D.J. Rankin, H. Kokko, Do males matter? the role of males in population dynamics, Oikos 116 (2) (2007) 335-348.

[24] K.H. Rosen, Mathematical models for polygamous mating systems, Math. Model. 4 (1983) 27-39.
[25] J.G. Skellam, Random dispersal in theoretical populations, Biometrika 38 (1951) $196-218$

[26] A. Trochet, D. Legrand, N. Larranaga, S. Ducatez, O. Calvez, J. Cote, J. Clobert, M. Baguette, Population sex ratio and dispersal in experimental, two-patch metapopulations of butterflies, J. Anim. Ecol. 82 (2013) 946-955.

[27] R.W. Van Kirk, M.A. Lewis, Edge permeability and population persistence in isolated habitat patches, Nat. Resour. Model. 12 (1) (1999) 37-64. 